\title{
PERMUTATIONS WITHOUT 3-SEQUENCES
}

\author{
JOHN RIORDAN
}

1. Introduction. The enumeration of permutations of $n$ distinct elements without rising 2 -sequences $12,23, \cdots, n-1 n$, is given by Whitworth [1], ${ }^{1}$ who gives also the enumeration when $n 1$ is added to this set of sequences. More recently, Kaplansky [2] and Wolfowitz [4] have enumerated permutations without rising or falling 2-sequences, that is, without $21,32, \cdots, n n-1$ as well as $12, \cdots, n-1 n$. An addition to these results, the enumeration of permutations without 3 -sequences, $123, \cdots ; n-2 n-1 n$ is given here. This case, aside from its general interest as a natural extension of its predecessors, has a particular interest because it is a relatively simple example of failure of what Kaplansky has called quasi-symmetry. In the method of inclusion and exclusion, or its symbolic equivalent, a case is said to be quasi-symmetric when the number of permutations having $k$ of the given properties is either zero or a function of $k$ alone.

2. The enumeration setting. Employing the symbolic method, with $q_{i j k}$ the probability that elements $i, j$ and $k$ are consecutive, the probability of finding a permutation without any of the 3 -sequences in question is

$$
P_{0}=\left(1-q_{123}\right)\left(1-q_{234}\right) \cdots\left(1-q_{n-2 n-1 n}\right) .
$$

The meaning of this is that on expansion a product of $q$ 's represents the probability of permutations having a particular set of 3 -sequences denoted by subscripts of these $q$ 's.

It is evident that the sequences chosen do not conflict; that is, it is possible to have any $k$ of the $n-2$ simultaneously.

For a single $q$ the number of permutations is $(n-3+1)$ !, for 3 elements are required for the corresponding 3 -sequence which may be permuted as a single entity; the corresponding probability is $(n-2) ! / n !$

For a product of two $q$ 's however, the case is otherwise. If the two are immediately adjacent, like 123,234 , the number of permutations is $(n-4+1)$ ! or $(n-3)$ !; if not, like 123,345 or 123,456 , the number is either $(n-5+1)$ ! or $(n-6+2)$ !, in either case $(n-4)$ !. If sequences are denoted by their initial numbers, the number of permutations is

Received by the editors May 25, 1945.

${ }^{1}$ Numbers in brackets refer to the Bibliography at the end of the paper. 
$(n-3)$ ! or $(n-4)$ ! according as the two initial numbers in rising order are a 2 -sequence or not.

In the same way, for three $q$ 's, the number of permutations with the corresponding three 3 -sequences is $(n-4) !,(n-5)$ ! or $(n-6)$ ! according as the set of three initial numbers in rising order has 2,1 or 02 -sequences, with a 3 -sequence counting as two 2 -sequences.

In general, for a product of $k q$ 's, the number of permutations with $k$ corresponding 3 -sequences is $(n-2 k+i)$ ! when the set of $k$ initial numbers in rising order has $i 2$-sequences or for euphony successions, a $j$-sequence counting as $j-1$ successions.

The enumeration is then completed by determining the number of combinations $k$ at a time of the given $n-23$-sequences which correspond to $i$ successions in the set of their $k$ initial numbers in rising order, as given by the following theorem:

THEOREM. The number of combinations of $n$ things $k$ at a time such that each combination in rising order has $i$ successions, a $j$-sequence counting as $j-1$ successions, is

$$
f_{i}(n, k)=C_{k-1, i} C_{n-k+1, k-i} \text {. }
$$

3. Proof of the theorem. The proof depends essentially on the recurrence in the following lemma : ${ }^{2}$

Lemma. $f_{i}(n, k)=f_{i}(n-1, k)+f_{i}(n-2, k-1)+f_{i-1}(n-1, k-1)$ $-f_{i-1}(n-2, k-1)$.

The combinations may be subdivided into those which do not contain $n$ and those which do, and the latter in turn into those which do not contain $n-1$ and those which do. The number without $n$ is $f_{i}(n-1, k)$. When $n$ is present and $n-1$ absent, $n$ is not in any succession, and the corresponding number is $f_{i}(n-2, k-1)$. When both $n$ and $n-1$ are present, there must be $i-1$ successions among the combinations of $n-1$ elements $k-1$ at a time which contain $n-1$. The combinations of $n-1$ elements $k-1$ at a time with $i-1$ successions is $f_{i-1}(n-1, k-1)$, the number of these which do not contain $n-1$ is $f_{i-1}(n-2, k-1)$, hence the number which do is $f_{i-1}(n-1, k-1)$ $-f_{i-1}(n-2, k-1)$.

Then the formula in the theorem may be derived, step by step, for successive values of $i$. The case $i=0$ has been given by Kaplansky

${ }^{2}$ This lemma replaces a succession of lemmas, for successive values of $i$, in an earlier version, the change being a response to suggestions from Irving Kaplansky, S. O. Rice, and C. E. Shannon. To Dr. Kaplansky I owe also a simplification of the procedure for verification of the formula in the theorem as well as the correction of an error in the variance formula (6) below. 
[3]; the recurrence

$$
f_{0}(n, k)=f_{0}(n-1, k)+f_{0}(n-2, k-1)
$$

which follows from the lemma with $f_{-1}=0$, and boundary conditions $f_{0}(n, 1)=n, f_{0}(k, k)=0, k>1$, lead to the formula in the theorem. In the general case the formula of the theorem must be checked for the lowest values of the other two variables of the induction, $n$ and $k$. The lowest value of $n$ is $k$ and the corresponding boundary relation is $f_{i}(k, k)=1, i=k-1$, and zero otherwise. The lowest value of $k$ is $i+1$ and $f_{i}(n, i+1)=n-i$.

4. Completed enumeration. These results may now be used to put (1) in an explicit form, as follows :

$$
P_{0}=\sum_{k=0}^{n-2}(-)^{k} \sum_{i=0}^{k-1} C_{k-1, i} C_{n-k-1, k-i} \frac{(n-2 k+i) !}{n !} .
$$

Note that in the present application of the theorem $n$ is replaced by $n-2$, the number of 3 -sequences in question.

Another writing of this result is

$$
P_{0}=\sum_{k=0}^{n-2}(-)^{k} a_{n, k}
$$

with

$$
\begin{aligned}
a_{n, k} & =\sum_{i=0}^{k-1} C_{k-1, i} C_{n-k-1, k-i} \frac{(n-2 k+i) !}{n !} \\
& =\sum_{i=0}^{k-1} C_{k-1, i} C_{n-k-1, i+1} \frac{(n-i-k-1) !}{n !} .
\end{aligned}
$$

This is a convenient form for writing the more general result for the probability that a permutation has $x 3$-sequences of the specified kind, which is

$$
P_{x}=\sum_{k=0}^{n-2}(-)^{k+x} C_{k, x} a_{n, k}
$$

with $C_{k, x}=0, k<x$, by a known process (Kaplansky [2]). Also the coefficients $a_{n, k}$ are related to $k$ th factorial moments $(m)_{k}$ of the distribution $P_{x}$ by

$$
k ! a_{n, k}=(m)_{k} .
$$

Hence the expected number of sequences $(m)_{1}$ is 


$$
(m)_{1}=m_{1}=a_{n, 1}=\frac{(n-2)(n-2) !}{n !}=\frac{n-2}{n(n-1)}
$$

and the variance is

(6)

$$
\begin{aligned}
V & =(m)_{2}+m_{1}-m_{1}^{2}=2 a_{n, 2}+a_{n, 1}-a_{n, 1}^{2} & \\
& =\frac{1}{(n)_{2}(n)_{3}}\left[n^{4}-3 n^{3}+n^{2}-6 n+8\right], & n>3,
\end{aligned}
$$

where $(n)_{i}$ is the factorial $n(n-1) \cdots(n-i+1)$.

A short table of numbers $n ! P_{x}$ follows.

\begin{tabular}{|c|c|c|c|c|c|c|c|c|}
\hline$n / x$ & 0 & 1 & 2 & 3 & 4 & 5 & 6 & 7 \\
\hline 3 & 5 & 1 & & & & & & \\
\hline 4 & 21 & 2 & 1 & & & & & \\
\hline 5 & 106 & 11 & 2 & 1 & & & & \\
\hline 6 & 643 & 62 & 12 & 2 & 1 & & & \\
\hline 7 & 4547 & 406 & 71 & 13 & 2 & 1 & & \\
\hline 8 & 36696 & 3046 & 481 & 80 & 14 & 2 & 1 & \\
\hline 9 & 332769 & 25737 & 3708 & 559 & 89 & 15 & 2 & 1 \\
\hline 10 & 3349507 & 242094 & 32028 & 4414 & 640 & 98 & 16 & 2 \\
\hline
\end{tabular}

Permutations With $x$ 3-SEQuences

Numbers $n ! P_{x}$

1. W. A. Whitworth, Choice and chance, London, 1901, pp. 102 and 104.

2. Irving Kaplansky, Symbolic solution of certain problems in permutations, Bull. Amer. Math. Soc. vol. 50 (1944) pp. 906-914.

3. - Solution of the "probleme des ménages," Bull. Amer. Math. Soc. vol. 49 (1943) pp. 784-785.

4. J. Wolfowitz, Additive partition functions and a class of statistical hypotheses, Annals of Mathematical Statistics vol. 13 (1942) pp. 247-279.

Bell Telephone Laboratories 